\title{
O SIGNIFICADO DA FISIOTERAPIA PARA CUIDADORES DE CRIANÇAS COM PARALISIA CEREBRAL
}

\section{THE MEANING OF PHYSIOTHERAPY FOR CAREGIVERS OF CHILDREN WITH CEREBRAL PALSY}

\author{
Marina Baía do Vale ${ }^{1}$ \\ Priscila Viegas Bessa ${ }^{2}$ \\ Taiane de Socorro Silva Natividade ${ }^{3}$ \\ Ivete Furtado Ribeiro Caldas ${ }^{4}$
}

\begin{abstract}
Resumo: O objetivo desse estudo é verificar o conhecimento geral do cuidador sobre paralisia cerebral (PC), os aspectos relacionados ao tratamento fisioterapêutico utilizado em crianças com PC, bem como a compreensão e a prática do cuidador na continuação em casa da fisioterapia. Trata-se de um estudo qualitativo em que foram entrevistados cinco cuidadores cujos filhos recebem tratamento fisioterapêutico para PC. Os resultados foram categorizados e discutidos de acordo com a temática, e mostraram a dificuldade do cuidador na compreensão da patologia e no tratamento fisioterapêutico de crianças com PC. Portanto, faz-se necessário maior interação entre fisioterapeuta-cuidador-paciente para oferecer mais orientações, esclarecer dúvidas e prestar assistência adequada às crianças com PC e aos seus cuidadores.
\end{abstract}

Palavras-chave: Paralisia Cerebral; Fisioterapia; Cuidadores; Metodologia qualitativa; Análise de conteúdo.

\begin{abstract}
The purpose of the study is to verify the caregiver's general knowledge about cerebral palsy (CP), the physical therapy treatment used in children with CP and the understanding of the caregiver about the physical therapy treatment at home for those children. It is a qualitative study obtained by content analysis. Five caregivers of children with CP that receive physiotherapy treatment were interviewed. The results were categorized and discussed according to the topics and they shown the difficulty of the caregiver in comprehend the patology and the physical therapy treatment in children with $\mathrm{CP}$. Therefore, it is necessary a better interaction between professional-caregiver-patient to offer more orientation, clarify doubts and provide adequate assistance to the children with CP and their caregivers.
\end{abstract}

Keywords: Cerebral Palsy; Physiotherapy; Caregivers; Qualitative methodology; Content analysis.

\footnotetext{
1 Mestranda em Saúde e Meio Ambiente pela Universidade Federal do Pará (UFPA). Fisioterapeuta. Residente em Saúde da Mulher e da Criança pela Universidade Federal do Pará (UFPA), Belém, Pará, Brasil. E-mail: mariivalee@ hotmail.com

${ }^{2}$ Fisioterapeuta graduada pela Universidade Federal do Pará (UFPA), Belém, Pará, Brasil. E-mail: p.viegas@yahoo.com.br

${ }^{3}$ Fisioterapeuta graduada pela Universidade Federal do Pará (UFPA), Belém, Pará, Brasil. E-mail: ts.natividade@gmail.com

${ }^{4}$ Doutora em Neurociências e Biologia Celular pela Universidade Federal do Pará (UFPA). Coordenadora do Laboratório de Desenvolvimento Infantil (LADIN) da Universidade do Estado do Pará (UEPA), Marabá, Pará, Brasil. E-mail: ivbeiro@yahoo.com.br
} 


\section{Introdução}

Os cuidadores de crianças com Paralisia Cerebral (PC), na maioria das vezes, assumem as principais responsabilidades a longo prazo, dispensando maior atenção no cuidado dessas criança em virtude das limitações funcionais ocasionadas pela PC (CHILUBA; MOYO, 2017). Também denominada encefalopatia crônica não progressiva da infância, a PC é descrita como um grupo de transtornos do movimento e da postura que acometem o sistema nervoso central (SNC) ainda em fase de desenvolvimento, resultando em alterações na estrutura e na função do sistema neuromusculoesquelético (GOMES et al., 2015).

A PC acomete cerca de 2 a cada 1000 crianças nascidas vivas no mundo, a prevalência tem se mantido estável nos últimos 10 anos, no entanto, há grande diferença epidemiológica entre países desenvolvidos e em desenvolvimento. Essa diferença é atribuída aos cuidados pré-natais e atendimento à gestante (BRASIL, 2013). No Brasil, estima-se que a cada 1.000 crianças nascidas vivas, sete são portadoras de PC, aumentando de 30.000 a 40.000 novos casos por ano (BRASIL, 2013; JACQUES, 2010).

Estudo aponta que a intervenção precoce pode ajudar crianças com alterações funcionais a alcançarem o desenvolvimento "ideal" para sua condição específica, proporcionando-nas e às suas famílias melhores níveis de estabilidade e segurança. No entanto, em graus avançados, a dependência funcional da criança acarreta sobrecarga física e psicológica aos familiares, principalmente às mães que mais frequentemente cuidam da criança. Essa dependência afeta negativamente a qualidade de vida dos cuidadores (CHESHIRE, BARLOW, POWELL, 2010).

Nesse sentido, o nascimento de filhos com limitações motoras e/ou cognitivas proporciona em seus cuidadores diversas modificações em suas vidas, como: alteração na vida profissional, cotidiano mais exaustivo, redução do tempo livre e, na maioria das vezes, dedicação exclusiva ao filho. Essas situações geram insatisfações, divergências de opinião, discussões e atritos entre as pessoas do seu convívio, em especial com o cônjugue que lida diretamente com essa situação estressante e desafiadora. Diante disso, sentimentos como depressão, angústia, medo, solidão, fuga e rejeição ou superproteção da criança fazem parte do cotidiano desses cuidadores (SILVA; RAMOS, 2014). Dessa forma, no processo de prestação de cuidados, as habilidades dos cuidadores de prover suas próprias necessidades emocionais, pessoais, físicas, sociais e financeiras são seriamente comprometidas (CHILUBA; MOYO, 2017). 
O estresse causado pela pessoa que assume o papel de cuidador é perceptível, pois assumem a carga horária total de uma série de ações de cuidado que demandarão mais tempo e atenção, fator que pode interferir na compreensão e dedicação dispensada ao tratamento fisioterapêutico dos seus filhos (SILVA; RAMOS, 2014). Todavia, a interação da família e do cuidado fisioterapêutico está aquém do esperado, pois a grande maioria dos cuidadores não apresenta o grau de informações necessárias sobre a PC e o tratamento fisioterapêutico, deixando-os menos participativos na reabilitação de seus filhos (MELLO; ICHISATO; MARCON, 2012).

Assim, o contato fisioterapeuta-paciente-família é essencial para um melhor prognóstico da criança e melhoria na qualidade de vida de seus familiares/cuidadores. Isso é possível através de interação satisfatória do profissional com a criança e a família. Essa relação é também importante para otimizar o tratamento domiciliar pelos cuidadores, pois, muitas vezes, eles devem continuar a fisioterapia em casa, e para isso, o cuidador precisa conhecer detalhadamente o tratamento e sua função no quadro evolutivo da criança (ALLEGRETTI et al., 2001). O conhecimento da condição e da importância da fisioterapia na reabilitação pode levar a melhora das funções motoras da criança, aperfeiçoando o seu prognóstico (OLAWALE; DEITH; YAADAR, 2013; DAMBI; JELSMA, 2014). Portanto, esse estudo objetiva verificar o conhecimento geral do cuidador sobre paralisia cerebral $(\mathrm{PC})$, os aspectos relacionados ao tratamento fisioterapêutico utilizado em crianças com PC, bem como a compreensão e a prática do cuidador na continuação em casa da fisioterapia.

\section{Método}

O estudo tem caráter predominantemente qualitativo, pois tem foco na compreensão de processos em uma dada população e na análise profunda de opiniões da mesma (TURATO, 2005; TRIVIÑOS, 1987). Foi aprovado pelo Comitê de Ética em Pesquisa da Universidade Federal do Pará (UFPA) (Parecer nº 850.614) em conformidade com as normas nacionais de ética e pesquisa envolvendo seres humanos de acordo com a resolução 466/12.

Participaram do estudo cinco cuidadores $(\mathrm{C} 1, \mathrm{C} 2, \mathrm{C} 3, \mathrm{C} 4, \mathrm{C} 5)$ de crianças com diagnóstico de PC que realizavam tratamento fisioterapêutico no Ambulatório de Desenvolvimento Infantil do Hospital Universitário Bettina Ferro de Souza (HUBFS) da Universidade Federal do Pará (UFPA). O número de participantes inclusos justifica-se 
pela metodologia escolhida, que visa compreender, interpretar, e descrever fenômenos e significados, justificando a casuísta da pesquisa ser limitada (TURATO, 2005; TRIVIÑOS, 1987). Foram inclusos cuidadores de crianças diagnosticadas com PC que realizavam tratamento fisioterapêutico regularmente no referido hospital há pelo menos três meses. Foram excluídos cuidadores menores de idade, cujos filhos apresentavam outra doença associada à $\mathrm{PC}$ ou que realizavam outros tratamentos fisioterapêuticos fora do ambiente hospitalar onde foi realizada a pesquisa.

O período de coleta de dados foi de Janeiro a Fevereiro de 2016 e o método de coleta de dados escolhido foi a entrevista semiestruturada, que consiste na utilização de um roteiro previamente elaborado, caracterizado como uma entrevista com perguntas básicas, apoiadas em teorias e hipóteses no tema da pesquisa, que fornecerá a descrição, explicação e compreensão dos fenômenos sociais (TURATO, 2005; TRIVIÑOS, 1987). Cinco perguntas foram elaboradas para a entrevista semiestruturada: O que você entende sobre PC? O que você entende sobre fisioterapia? Qual a sua opinião em relação a criança depois que ela iniciou a fisioterapia, ela obteve melhora, piora, no quê? Quanto tempo você acha que a criança deve continuar fazendo fisioterapia? O que você aprendeu assistindo as sessões de fisioterapia, é possível fazer com a criança em casa?

Inicialmente, foi realizado um levantamento dos prontuários das crianças que se enquadravam nos critérios de inclusão, posteriormente, os pesquisadores entravam em contato com seus respectivos cuidadores durante as sessões de fisioterapia quando eram explicados os procedimentos da pesquisa. Aqueles que aceitavam participar da pesquisa e assinavam o TCLE, um outro encontro era agendado para preenchimento da ficha de cadastro que continha os dados pessoais e sociodemográficos do cuidador e da criança, além dos dados pertinentes à fisioterapia (quanto tempo a criança realizava, se era somente no ambiente ambulatorial ou se já havia realizado algum outro tipo de tratamento fisioterapêutico). Nesse mesmo dia também era realizada a entrevista semiestruturada com o cuidador que acontecia em uma sala reservada ao lado do ambulatório onde as crianças realizavam as sessões de fisioterapia.

As coletas foram realizadas no turno matutino e/ou vespertino, respeitando o horário de atendimento fisioterapêutico das crianças e/ou a disponibilidade dos participantes. Os dados coletados por meio de entrevistas foram gravados em um gravador e realizadas somente uma vez com cada cuidador, com duração média de 30 minutos.

A análise e interpretação dos dados foi realizada através da análise de conteúdo, visando utilizar a categorização, descrição e interpretação como etapas essenciais da 
análise dos dados (MORAES, 1999; MINAYO, 2002 ). Ao analisar os dados qualitativos, os resultados registrados foram transcritos e organizados em temas principais respeitando as perguntas da entrevista semiestruturada. Os princípios da análise narrativa foram usados para construir uma narrativa das necessidades e experiências dos cuidadores.

\section{Resultados/Discussão}

Os resultados obtidos através das entrevistas foram organizados e discutidos de acordo com a ordem das perguntas realizadas na pesquisa, sendo as falas dos cuidadores categorizadas e agrupadas de acordo com a temática.

\subsection{Conhecimento acerca da doença}

A declaração a seguir mostra que o cuidador não tem conhecimento sobre a PC, assumindo, inclusive, não entender por ter nível baixo de escolaridade:

É um meio que a gente não pode explicar, só o neurologista mesmo pode explicar. Até porque eu tenho a série baixa, não entendo muito as coisas (C3).

Esse resultado mostra a dificuldade do cuidador em compreender sobre a patologia do seu filho, associando tal dificuldade a complexidade do assunto e ao baixo nível de escolaridade. Nesse contexto, a aceitação de uma criança com PC leva a um processo de reestruturação familiar que depende, em grande parte, de como os pais entendem o diagnóstico, atribuindo a ele um significado para suas vidas e de seu filho, sendo, portanto, necessário o entendimento dos pais e cuidadores a respeito da doença. Todavia, a maioria das famílias com crianças com PC presentam baixo nível de escolaridade, o que contribui para a dificuldade do entendimento acerca da doença (OLAWALE; DEIH; YAADAR, 2013).

Outro afirmou que ainda não havia discutido acerca do assunto: Eu não sei, porque ainda não falaram (C5).

Nessa declaração do cuidador denota uma interação frágil entre o fisioterapeuta e e o cuidador em relação ao entendimento deste sobre a patologia do seu filho. Estudos reforçam que o conhecimento da condição clínica e funcional pode levar a melhorias nas funções motoras da criança, levando assim a um melhor prognóstico (OLAWALE; DEITH; YAADAR, 2013; DAMBI; JELSMA, 2014). Portanto, é função do fisioterapeuta e de qualquer profissional da área da saúde dar esclarecimentos aos cuidadores, 
reforçando a importância do cumprimento das orientações desses profissionais (SARI; MARCON, 2008).

Houve ainda um cuidador que demonstrou negação em relação a doença do seu filho(a):

A minha filha não tem essa doença, não... Ela é normal, a única coisa que ela não faz é andar (C1).

Este resultado reflete o sentimento de negação em relação a doença que também é esperado nesse contexto. O nascimento de um bebê especial gera nos pais uma gama de reações diante do inesperado, que incluem períodos de crises emocionais e de adaptações psicossociais, tornando-os susceptíveis ao desenvolvimento de problemas relacionados à depressão, fuga, negação da doença, rejeição ou superproteção da criança (RIBEIRO; MORAES; BELRAME, 2008). Dessa forma, quanto mais precoce for realizada a intervenção na criança maior serão os benefícios motores e funcionais que a mesma obterá, além de beneficiar o cuidador nesse processo de aceitação e comprometimento com o tratamento (CARVALHO, 2010).

Outros, contudo, demonstram conhecimento prévio a respeito da doença, um por ter experiência com outro familiar:

Olha, em questão... Não foi uma coisa nova para mim porque eu tinha uma prima que ela também tinha Paralisia Cerebral só que ela era mais crítica que o meu filho (...) (C2).

E outro por entender a causa da doença:

A explicação que eu tenho é que ele tem paralisia cerebral, que é algo que foi atingido na gravidez dela né, devido ela não ter se cuidado, não ter feito prénatal, ai atingiu ele pela aquela doença chamada de toxoplasmose, que é... que é pelo animal, e acabou atingindo o cérebro dele, as partes do olho direito dele e ai ele ficou assim (C4).

\subsection{Entendimento sobre a Fisioterapia}

Quando questionados sobre o que era fisioterapia, a resposta dos cuidadores foi unanime afirmando ser algo para melhorar a qualidade de vida da criança com paralisia cerebral:

A fisioterapia é bom que só, ela já tá até dando uns passinhos já, já levanta, já anda um pouquinho. Pra mim foi bem importante, ela precisa ainda muito da fisioterapia (C1).

Outros cuidadores enfatizaram o impacto da fisioterapia como ferramenta para melhorar a coordenação motora das crianças:

Que é pra melhorar a coordenação motora dele (C2). 
Fazer os movimentos dele pra ele andar, pegar alguma coisa, que ele pega pouco (C5).

O contexto de estimulação também foi abordado pelos cuidadores:

Olha, a fisioterapia eu sei que é uma coisa boa, né? Que serve pra estimular, serve pro desenvolvimento dele, né? (C3).

Olha, a fisioterapia no caso é pra ajudar né, pra cada vez mais estimular (C4).

Tais resultados indicam que todos os cuidadores percebem a fisioterapia como algo importante para seus filhos, tanto para o desenvolvimento motor, quanto para o desenvolvimento da coordenação motora, destacando também a função da fisioterapia como estimulação precoce. Além do mais, os cuidadores também reconhecem a importância da fisioterapia na recuperação de seus filhos.

A marcha e a coordenação motora são muitas das vezes as maiores dificuldades apresentadas em crianças com PC e fonte de preocupação para os cuidadores, como foi destacado nas declarações da maioria dos cuidadores. O tratamento fisioterapêutico em crianças com PC realiza intervenções motoras que têm como objetivo melhorar a flexibilidade, facilitar padrões normais de movimento e melhorar as capacidades motoras básicas para um melhor desempenho nas habilidades funcionais dessas crianças, podendo então, estimular o desenvolvimento motor e coordenação motora das mesmas (OLAWALE; DEIH; YAADAR, 2013).

\subsection{O tratamento fisioterapêutico na qualidade de vida da criança com PC}

Quando entrevistados sobre a melhora da criança após o início do tratamento fisioterapêutico, os cuidadores afirmaram unanimemente um impacto positivo da fisioterapia na qualidade de vida das crianças, principalmente em ganhos motores de marcha e independência funcional:

Ajudou, depois que ela começou a fazer fisioterapia ela já até se movimenta mais, ela já até procura já andar, ela já se levanta, ela já tá dando uns passinhos (C1).

Que ele melhorou. Dá pra ver perfeitamente o quanto ele melhorou. Porque antigamente ele não se mexia muito na cama, ele ficava todo "paradão". Quando ele conseguia virar de costas ele não conseguia voltar. Agora não, ele se mexe, ele desce da cama. Agora que ele começou a engatinhar. Ele tá bem espertinho (C2).

Ele começa a pegar as coisas com uma mão, ai já tá sentando e se arrastando só ele (C5). 
Essas declarações dos cuidadores demonstram que a fisioterapia é essencial para a reabilitação das crianças com $\mathrm{PC}$, pois promove avanços importantes na evolução clínica de seus filhos. O tratamento fisioterapêutico para crianças com PC tem foco o desenvolvimento neurofisiológico, biomecânico, sensorial, a educação condutiva e o uso de dispositivos ortopédicos. Várias técnicas podem ser utilizadas pela fisioterapia no tratamento de crianças com PC, dentre elas: técnicas de terapia manual, mobilizações, alongamentos, estimulação elétrica, treino de deambulação e de atividades de vida diária (BRANDÃO et al., 2015; LIN et al., 2011; LOI et al., 2015; FERRE et al., 2015; POOL et al., 2015).

Uma revisão sistemática da literatura mostrou que o prognóstico motor em crianças com PC está associado, entre outros fatores, à assistência terapêutica oferecida ao paciente. O desempenho motor da criança muda conforme ela recebe tratamento de uma equipe de reabilitação, incluindo fisioterapeutas, terapeutas ocupacionais, psicólogos, médicos, entre outros (REBEL et al., 2010). Todavia, o nível de funcionalidade da criança, sua participação e envolvimento na atividade e nas situações do cotidiano também contribuem para aumento do estado geral de saúde e qualidade de vida (LAW et al., 2006; IMMS et al., 2008; SIMSEK; TUC, 2014).

\subsection{O período do tratamento fisioterapêutico na criança com PC}

Quando questionados a respeito de quanto tempo a criança deveria permanecer em tratamento fisioterapêutico, os cuidadores responderam não saber exatamente o período. Um deles afirmou que a criança deveria aprender a andar antes do tratamento ser finalizado:

Eu acho que por uns 4 ou 5 anos ele ainda vai permanecer ainda sim. Até ele aprender a andar... (C1).

Enquanto os outros afirmaram não saber por não terem conhecimento acerca do futuro, e de como a criança responderia ao tratamento:

Isso daí é o tempo que vai responder, né? (C3).

Olha... no caso aqui ele já tá pra sair, porque ele já vai fazer 6 anos, é... eu não tenho uma base pra te dizer quanto tempo ele vai fazer, porque isso depende muito também dele né, quanto ele vai suportar, se ele vai reagir bem, entendeu? Então o tempo eu não posso te responder (C5).

Esses resultados mostram a incerteza dos cuidadores quanto ao tempo de permanência dos seus filhos na fisioterapia. Estudo aponta que o período do tratamento fisioterapêutico na criança com PC é indeterminado, pois, cada criança responde de uma 
forma ao tratamento (DAMBI; JELSMA, 2014). A redução da incapacidade e melhora de função são metas de um programa de reabilitação fisioterapêutico, sendo o mesmo não finalizado quando a criança consegue andar, pois, em alguns casos, o déficit muscular e neurológico na criança com PC a impede de alcançar o desenvolvimento da marcha. A reabilitação é realizada até que a criança obtenha capacidade funcional para ser independente ou semi-independente. Portanto, o período de reabilitação é indeterminado, podendo ser recomendado pela vida inteira do paciente (DOMENECH et al., 2016).

\subsection{A continuidade da fisioterapia em casa}

Nesta pergunta, a maioria dos cuidadores respondeu saber como fazer alguns exercícios com a criança em casa:

Aprendi. Tem uns exercícios que a médica fala pra gente fazer em casa (C1).

Já aprendi alguns exercícios e a fazer, a gente faz o exercício mesmo e usa alguns aparelhos (C3).

A continuidade da fisioterapia em casa é extremamente necessária para um bom prognóstico do paciente com PC. Semelhante aos resultados do nosso estudo, um estudo recente mostrou efeitos positivos da fisioterapia em combinação com exercícios domiciliares relatando melhora no desempenho funcional e independência, aquisição de novas habilidades e melhor desempenho motor global (DOMENECH et al., 2016). A presença dos cuidadores e sua participação no tratamento fisioterapêutico das crianças tem como objetivo principal a continuidade e a manutenção, no domicilio, da preservação do alinhamento biomecânico, da manutenção muscular e estímulo da interação cuidadorfilho (CARVALHO et al., 2010; BRASIL, 2013).

Um estudo qualitativo exploratório e descritivo, realizado com mães de crianças menores de cinco anos com PC, investigou a participação dos pais na reabilitação de seus filhos e suas expectativas em relação aos resultados do tratamento. Os autores destacam que as mães que receberam várias sessões de aconselhamento sentiram-se mais seguras sobre a realização de exercícios fisioterapêuticos em casa. A fisioterapia combinada com exercícios domiciliares contribui ainda mais para a recuperação dos pacientes com alterações físicas e funcionais (SARI; MARCON, 2008).

Porém, pode-se perceber também a falta de conhecimento na importância de dar continuidade do tratamento em casa, além do medo e da insegurança na realização dos exercícios pelos mesmos:

Mais ou menos... fazer uns movimentos na perna dele, quando eu faço... (C5). 
O fisioterapeuta ensina a mãe pra fazer em casa. Só que não é o correto porque a gente não sabe fazer direito, entendeu? Aí se a gente faz o movimento repetitivo errado pode vir a trazer danos à criança, então eu deixo pra doutora mesmo (C2).

Como mencionado nas declarações acima, a fragilidade da criança é algo que assusta o cuidador, pois estes têm medo de errar e demonstram insegurança em dar continuidade do tratamento em casa (LIN et al., 2011). Outro fator que impede a continuidade do tratamento em domicílio é a falta de orientação ao cuidador (MILBRATH et al., 2008). Esse descrédito dado por alguns profissionais faz com que os membros da família possam se sintir desvalorizados, criando sentimentos negativos em relação à rede de apoio que deveria cuidar de seus filhos. Nesse sentido, a percepção da dificuldade de alguns cuidadores em aderir e participar do tratamento fisioterapêutico tem suscitado grandes dúvidas relacionadas aos benefícios do tratamento domiciliar, uma vez que a adesão dos mesmos no tratamento é crucial no processo de evolução da criança (RIBEIRO; MORAES; BELTRAME, 2008).

Contudo, para minimizar a fragilidade da interação fisioterapeuta-cuidadorcriança devem ser englobadas no tratamento fisioterapêutico de crianças com PC orientações familiares e estimulações afetiva-emocional a partir de habilidades potenciais da criança. As trocas de informações resultantes da interação fisioterapeuta/família devem estar bem estabelecidas para a definição dos objetivos funcionais, possíveis na realidade motora, cognitiva e social em que a criança se encontra. Essas trocas contribuirão para a superação das dificuldades vivenciadas diariamente no relacionamento dessa família com a criança (MELLO; ICHISATO; MARCON, 2012).

Além do uso de técnicas específicas, o tratamento fisioterapêutico também deve enfatizar a necessidade de estratégias de educação em saúde e o fornecimento de informações sobre o diagnóstico, o tratamento e o prognóstico. Em um estudo, pesquisadores entrevistaram pais de crianças com Síndrome de Down, e os resultados mostraram que eles apresentam muitas dúvidas sobre a condição clínica de seus filhos (RIBEIRO; BARBOSA; PORTO, 2011). Outra pesquisa investigou a influência da oferta de orientações fisioterapêuticas em cuidadores de crianças com PC e encontrou melhora no desenvolvimento neurológico das crianças, afirmando que o envolvimento dos pais contribui diretamente na evolução do prognóstico de seus filhos (PALIGA; COSTA, 2005).

Entretanto, muitas vezes as recomendações dadas pelos profissionais de saúde, incluindo o fisioterapeuta, não podem ser sempre seguidas inteiramente devido à 
sobrecarga de papel do cuidador. Dessa forma, esses profissionais precisam abordar os cuidadores de forma diferente de acordo com suas necessidades. Eles devem conhecer outros aspectos da criança a ser tratada, a fim de prestar assistência individualizada aos seus cuidadores, tornando o tratamento mais completo, e assim contribuir para a evolução clínica e da qualidade de vida das crianças submetidas à reabilitação (BALTON; DUPAS, 2013).

\section{Considerações finais}

A interação entre fisioterapeutas e familiares de crianças com PC é de suma importância, pois ajuda superar as dificuldades associadas às limitações físicas da criança e criar um vínculo de confiança e segurança, proporcionando aos cuidadores a oportunidade de compartilhar sentimentos, necessidades e dúvidas. Essa abordagem diferenciada pode estimular e fortalecer o vínculo pai-mãe-filho, aumentar a compreensão e a aceitação da doença e promover uma maior conscientização sobre a importância da participação ativa de todos os membros da família no tratamento.

Em nosso estudo a análise das entrevistas indica que a maioria dos cuidadores têm uma percepção positiva do tratamento fisioterapêutico, além de valorizarem e reconhecerem os benefícios do tratamento. Todavia, tanto os fisioterapeutas quanto outros profissionais da área da saúde que trabalham com reabilitação infantil devem focar a atenção aos cuidadores que não aderem aos programas de tratamento, em especial, no domicílio. Eles devem tentar identificar os motivos de sua não conformidade, oferecer orientações, aconselhamento e assistência para esclarecer dúvidas e ajudar a resolver dificuldades enfrentadas pelos cuidadores.

Diante disso, o presente estudo demonstra uma necessidade maior de interação profissional-cuidador-paciente para a melhoria da qualidade de vida de crianças com PC. O fisioterapeuta deve ser um profissional também provedor de conhecimento para seus pacientes e cuidadores, nesse caso, deve auxiliar o cuidador a entender acerca da doença e seu desenvolvimento, além de explicar o tratamento fisioterapêutico e ensinar a praticar na clínica/ambulatório os exercícios e técnicas a serem realizadas pelo cuidador para dar continuidade ao tratamento fisioterapêutico da criança em casa.

Entretanto, algumas limitações em nosso estudo devem ser destacadas. Durante a análise dos dados foi notado que algumas perguntas da entrevista semiestruturada poderiam ter sido elaboradas de uma forma mais esclarecedora para evitar respostas 
comuns e repetitivas dos entrevistados. Por exemplo, no contexto do entendimento acerca da fisioterapia no tratamento da criança com PC, algumas declarações dos cuidadores tornaram-se repetitivas por falta de esclarecimento na questão perguntada.

Recomenda-se que futuros estudos qualitativos analisem a relação fisioterapeutacuidador-paciente com o intuito de entender quais aspectos relacionados a qualidade de vida da criança com PC podem ser modificados baseados na relação do profissional com o cuidador. E finalmente, estudos qualitativos adicionais sobre a percepção da criança em fisioterapia são sugeridos para complementar os dados deste trabalho.

\section{Referências}

ALLEGRETI, A. L. C. et al. A relação e o estabelecimento de vínculo entre terapeuta e criança com atendimento em instituição e clínica particular. Cadernos de Pós-Graduação em Distúrbios do Desenvolvimento, São Paulo, v. 1, n. 1, p.35-49. 2001.

BALTOR, M. R. R.; DUPAS, G. Experiências de famílias de crianças com paralisia cerebral em contexto de vulnerabilidade social. Rev Latino-Am Enfermagem, Ribeirão Preto, v. 21, n. 4, p.1-8, abr./jun. 2013.

BRANDÃO, M. et al. Hand use at home and clinical settings by children with cerebral palsy: a qualitative study. Occup. Ther. Int., Londres v.22, n. 1 p.43-50, out. 2015.

BRASIL. Ministério da Saúde. Secretaria de Atenção à Saúde. Departamento de Ações Programáticas Estratégicas. Diretrizes de atenção à pessoa com paralisia cerebral. Brasília: Ministério da Saúde, 2013.

CARVALHO, J. T. M. et al. Qualidade de vida das mães de crianças e adolescentes com paralisia cerebral. Fisioter. Mov., Curitiba, v. 23, n. 3, p. 389-397, jul./set. 2010.

CHESHIRE, A.; BARLOW, J.H.; POWELL, L.A. The psychosocial well-being of parents of children with cerebral palsy: a comparison study. Disabil Rehabil, Londres, v. 32, n. 20, p.1673-1677, jan. 2010.

CHILUBA, B. C.; MOYO, G. Caring for a cerebral palsy child: a caregiver's perspective at the University Teaching Hospital, Zambia Brian. BMC Res Notes, Berlin, v.10, n. 1, p.1-8, dez. 2017.

DAMBI, J. M.; JELSMA, J. The impact of hospital-based and community-based models of cerebral palsy rehabilitation: a quasi-experimental study. BMC Pediatrics, Londres, v.14, n. 1, p.1-10, dez. 2014.

DOMENECH, A. C. P. et al. Cerebral palsy: the meaning of physical therapy for mother caregivers. Fisioterapia em Movimento, Curitiba, v. 29, n. 4, p.757-766, dez. 2016.

FERRE, C. L. et al. Feasibility of caregiver-directed home-based hand-arm bimanual intensive training: A brief report. Dev Neurorehabil, Londres, v. 18, n. 1, p.69-74, set. 2015. 
GOMES, R. C. N. T. et al. Efeitos do treinamento resistido na força de indivíduo com paralisia cerebral. Revista Brasileira de Prescrição e Fisiologia do Exercício, São Paulo, v. 9, n. 15, p. 545-554, set./out. 2015.

IMMS, C. et al. Diversity of participation in children with cerebral palsy. Developmental Medicine \& Child Neurology, Londres v.50, n. 5, p. 363-369, mar. 2008.

JACQUES, K.C et al. Effectiveness of the hydrotherapy in children with chronic encephalopathy no progressive of the childhood: a systematic review. Rev Fisioterapia em Movimento, Curitiba, v. 23, n. 1, p. 53-61, jan./mar. 2010.

LAW, M. et al. Patterns of participation in recreational and leisure activities among children with complex physical disabilities. Developmental Medicine \& Child Neurology, Londres, v.48, n. 5, p.337-342, mar. 2006.

LIN, K. C. et al. Effects of home-based constraint-induced therapy versus dose-matched control intervention on functional outcomes and caregiver well-being in children with cerebral palsy. Research in Developmental Disabilities, Oxford, v.32, n. 5, p.1483-1491, set./out.. 2011.

LOI, E. C. et al. Myofascial structural integration therapy on gross motor function and gait of young children with spastic cerebral palsy: a randomized controlled trial. Front. Pediatr., Lausana, v. 3, n. 74, p. 1-10, set. 2015.

MELLO, R.; ICHISATO, S. M. T.; MARCON, S. S. Family perception on the disease and physiotherapeutic care of individuals with cerebral palsy. Rev Bras Enferm., Brasília v. 65, n. 1, p.104-109, jan./fev. 2012.

MILBRATH, V. M et al. Ser mulher mãe de uma criança portadora de paralisia cerebral. Acta Paul Enfer., São Paulo, v. 21, n. 3, p.427-31. 2008.

MINAYO, M. C. S. et. al. Pesquisa social: Teoria, método e criatividade. 21. ed. Petrópolis. Editora Vozes. 2002.

MORAES, R. Análise de conteúdo. Revista Educação, Porto Alegre, v. 22, n. 37, p.7-32. 1999.

OLAWALE, O. A.; DEIH, A. N.; YAADAR, R. K. Psychological impact of cerebral palsy on families: The African perspective. J Neurosci Rural Pract., Mumbai, v. 4, n. 2, p.159-163, abr. 2013.

PALIGA, C. S.; COSTA, J. C. Orientações fisioterapêuticas aos cuidadores de crianças portadoras de Paralisia Cerebral. Rev Medica HSVP., Passo Fundo, v. 17, n. 37, p.21-24, 2005.

POOL, D. et al. The orthotic and therapeutic effects following daily community applied functional electrical stimulation in children with unilateral spastic cerebral palsy: a randomized controlled trial. BMC Pediatrics., Londres, v. 15, n. 1, p. 1-10, out. 2015.

REBEL, M. F. et al. Prognóstico motor e perspectivas atuais na paralisia cerebral. Rev Bras Crescimento Desenvolv Hum., São Paulo v. 20 n. 2, p. 342-50, ago. 2010.

RIBEIRO, M.F.M., BARBOSA, M.A., PORTO, C.C. Paralisia cerebral e síndrome de Down: nível de conhecimento e informação dos pais. Cienc Saude Coletiva, Rio de Janeiro, v.16, n.4, p. 2099-2106, out. 2011. 
RIBEIRO, J.; MORAES, M. V. M.; BELTRAME, T. S. Tipo de atividade e relação interpessoal estabelecida entre fisioterapeuta e criança com paralisia cerebral no contexto de intervenção fisioterapêutica. Dinamys, Blumenau, v. 14, n. 1, p.89-95. 2008.

SARI, F. L.; MARCON, S. S. Participation of the family in physical therapy treatment of children with cerebral palsy. Rev. Bras Crescimento Desenvolv Hum., São Paulo, v. 18, n. 3, p. 229-239. 2008.

SILVA, C. C. B.; RAMOS, L. Z. Reação dos familiares frente à descoberta da deficiência do filho. Card. Terap. Ocup., São Carlos, v. 22, n. 1, p.15-23, out. 2014.

ŞIMSEK, T. T.; TUG, G. Examination of the relation between body mass index, functional level and health-related quality of life in children with cerebral palsy. Turkish Archives of Pediatrics, Istambul, v. 49, n. 2, p.130-137, jun. 2014.

TURATO, E. R. Métodos qualitativos e quantitativos na área da saúde: definições, diferenças e seus objetos de pesquisa. Rev Saúde Pública., São Paulo, v. 39, n. 3, p.507-514. 2005.

TRIVIÑOS, A. N. S. Introdução à pesquisa em ciências sociais: a pesquisa qualitativa em educação. 1.ed. São Paulo: Atlas, 1987.

Recebido em: 01 de março de 2018.

Aceito em: 24 de outubro de 2018. 